\title{
Microglobule formation and a microscopic order parameter monitoring the phase transition of aqueous poly $(N$-isopropylacrylamide) solution
}

\author{
Keiichi Yanase, ${ }^{1}$ Richard Buchner, ${ }^{2, *}$ and Takaaki Sato ${ }^{1, \dagger}$ \\ ${ }^{1}$ Department of Chemistry and Materials, Faculty of Textile Science and Technology, Shinshu University, Ueda, Nagano 386-8567, Japan \\ ${ }^{2}$ Institut für Physikalische und Theoretische Chemie, Universität Regensburg, D-93040 Regensburg, Germany
}

(Received 7 April 2018; revised manuscript received 26 June 2018; published 7 August 2018)

\begin{abstract}
The coil-to-globule transition of poly( $N$-isopropylacrylamide) (pNIPAm) in water is generally believed to be driven by hydrophobic interaction between the isopropyl groups of its side chains. However, it is still unclear how dehydration and critical fluctuations of the polymer chains are correlated. Here, we use small- and wide-angle $\mathrm{x}$-ray scattering and dielectric relaxation spectroscopy to cover a wide range of the relevant length and time scales, enabling us to grasp an overall picture of this phase transition. We find that the hydration number of pNIPAm decreases only moderately with temperature up to about $6 \mathrm{~K}$ below its spinodal temperature $T_{\mathrm{S}}$, but then drops steeply on approaching $T_{\mathrm{S}}$. This rapid dehydration is coupled to a mean-field-like power-law divergence of the correlation length $\xi$, representing fluctuations of the density order parameter. Real-space decoding of an observed interference peak reveals partial-globule formation even far below $T_{\mathrm{S}}$ and demonstrates that the polymer-rich phase above $T_{\mathrm{S}}$ can be understood as a high-density assembly of the microglobules. Strikingly, condensation of the microglobules and the divergence of $\xi$ do not run parallel. Instead, the condensation occurs only above $T_{\mathrm{S}}$ and is completed about $6 \mathrm{~K}$ above $T_{\mathrm{S}}$. The local number density of the microglobules, exhibiting a steplike increase just above $T_{\mathrm{S}}$, should be identified as an additional microscopic order parameter governing the phase transition of pNIPAm.
\end{abstract}

DOI: 10.1103/PhysRevMaterials.2.085601

\section{INTRODUCTION}

Phase transitions and critical phenomena in polymer solutions have a host of implications for the self-organization of amphiphilic molecules [1], the collapse of gel networks [2,3], and protein folding [4]. Poly( $N$-isopropylacrylamide) (pNIPAm) is among the most studied thermoresponsive polymers, undergoing a coil-to-globule transition in water at a temperature of approximately $32-34{ }^{\circ} \mathrm{C}$ [5-12]. For several decades, hydrophobic interaction has been considered to be the major cause for this collapse of the hydrophobic polymer chain in water.

More detailed mechanisms have been discussed in the literature. ten Wolde and Chandler claimed that solvent fluctuationinduced dewetting is an underlying mechanism of the coilto-globule transition of an ideal hydrophobic polymer chain [13]. Tanaka et al. proposed a cooperative-hydration picture, in which sequential hydrogen bond formation along a pNIPAm chain, triggered by cooperative interactions between nearestneighbor bound water molecules, explains its phase diagram $[14,15]$. Bischofberger et al. argued that it is the mean energetic state of the aqueous medium that determines hydrophobic hydration in aqueous pNIPAm [16].

Because of unfavorable $x$-ray scattering contrast, the static structure of pNIPAm was mostly studied by small-angle neutron scattering (SANS) in $\mathrm{D}_{2} \mathrm{O}$ solvent. Shibayama et al. [17] showed that SANS intensities of pNIPAm in $\mathrm{D}_{2} \mathrm{O}$ solvent recorded for $q \leqslant 2 \mathrm{~nm}^{-1}$ can be described by a single

\footnotetext{
*richard.buchner@chemie.uni-regensburg.de

†takaakis@shinshu-u.ac.jp
}

Ornstein-Zernike (OZ) equation, where $q$ is the magnitude of the scattering vector. Using SANS, Meier-Koll et al. [18] discussed values of the critical exponents describing the critical fluctuation. The high- $q$ deviation from the $\mathrm{OZ}$ behavior in the SANS intensities of pNIPAm was tentatively attributed to domain formation. Although it was pointed out that the transition temperature of pNIPAm in $\mathrm{D}_{2} \mathrm{O}$ is $1-2^{\circ} \mathrm{C}$ higher than in $\mathrm{H}_{2} \mathrm{O}$ [19-21], possible deuterium isotope solvent effects on its critical behavior remain rather ambiguous.

Hydration effects and dynamics of pNIPAm have been extensively studied by spectroscopic techniques [22-32]. Dehydration behavior during the phase transition was investigated by dielectric relaxation spectroscopy (DRS), although the number of experimental data points are limited $[24,28]$. A recent Raman multivariate curve resolution (Raman-MCR) study of Mochizuki and Ben-Amotz revealed that significant changes in the $\mathrm{OH}$-band frequency range of the spectrum occur only above the cloud-point temperature, where the pNIPAm chains collapse [32]. This unexpected observation appears to be inconsistent with the solvent-fluctuation induced transition mechanism.

Difficulty in reaching a general consensus regarding the counterintuitive phase behavior of aqueous pNIPAm has mostly arisen from the lack of microscopic insights into the coil and globule states. An electron paramagnetic resonance study on pNIPAm-based hydrogels demonstrated the coexistence of hydrophilic and hydrophobic environments over a wide temperature range and suggested that microphase separation takes place in coexisting swollen and collapsed regions during the collapse process [25]. However, the structural details and the role played by the swollen and collapsed domains in 

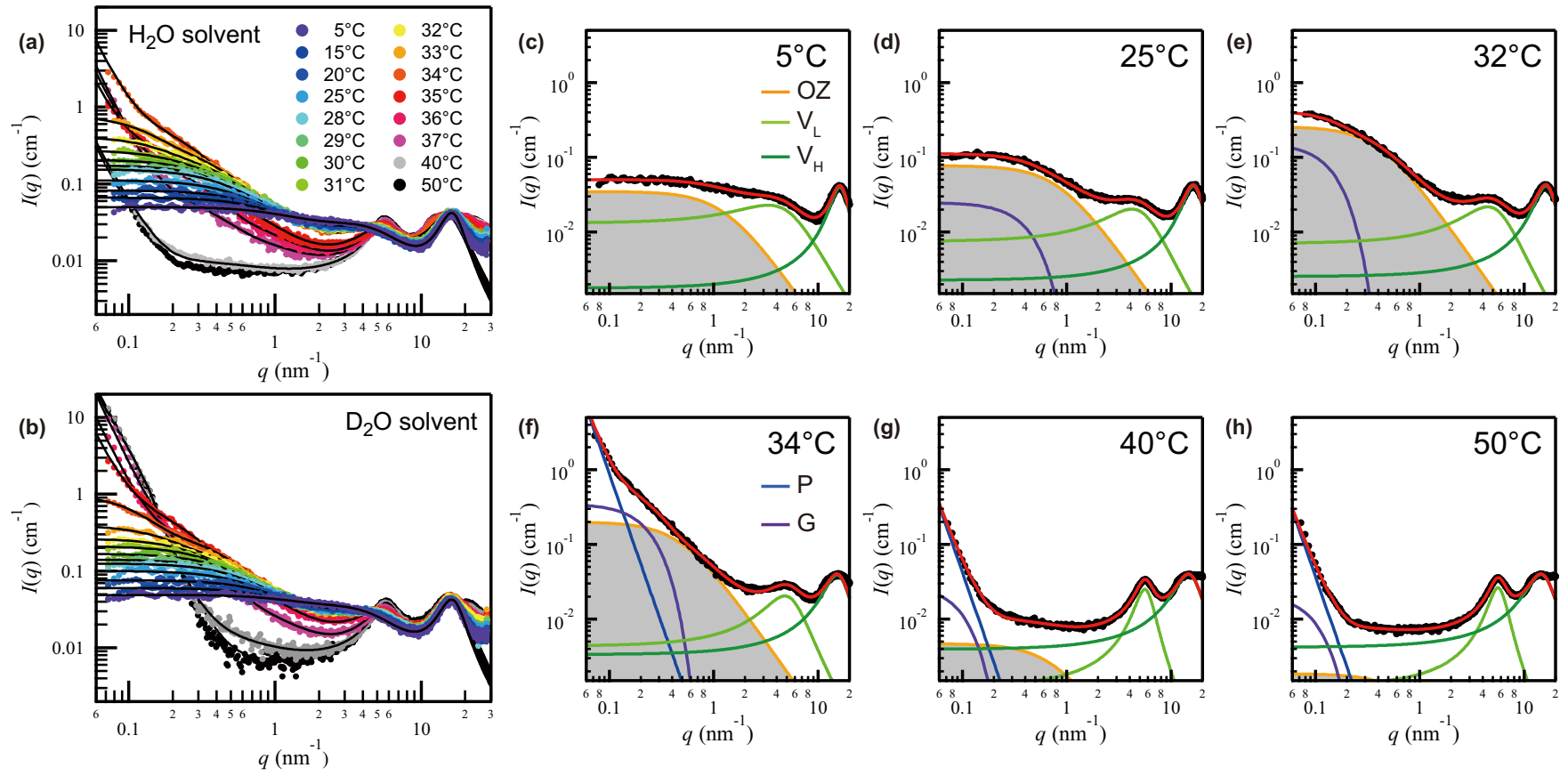

FIG. 1. Absolute SWAXS intensities $I(q)$ of $w=0.13$ pNIPAm weight fraction in (a) $\mathrm{H}_{2} \mathrm{O}$ and (b) $\mathrm{D}_{2} \mathrm{O}$ as a function of $T$ in $5 \leqslant T /{ }^{\circ} \mathrm{C} \leqslant 50$. (c)-(h) Full descriptions of $I(q)$ in $\mathrm{H}_{2} \mathrm{O}$ at 5, 25, 32, 34, 40, and $50^{\circ} \mathrm{C}$ based on superpositions of several scattering components [Eqs. (1)-(4)]. The full descriptions of $I(q)$ in $\mathrm{D}_{2} \mathrm{O}$ are provided in the Supplemental Material [36].

the phase transition still remain unclear. Furthermore, although the cooperative-hydration picture predicted partial globule formation even in the one-phase region [14,15], this has not yet been experimentally evidenced. To overcome all these issues, we study the phase transition of semidilute solutions of pNIPAm $\left(M_{\mathrm{W}} 29 \mathrm{kDa}\right)$ by means of simultaneous small- and wide-angle $\mathrm{x}$-ray scattering (SWAXS) and dielectric relaxation spectroscopy (DRS).

\section{METHODOLOGY}

Poly( $N$-isopropylacrylamide) (pNIPAm) having a nominal molecular weight of 19-30 kDa was purchased from Aldrich and was used as received. pNIPAm solutions with polymer weight fraction $w=0.13$ were prepared by dissolving the polymer in Millipore water $\left(\mathrm{H}_{2} \mathrm{O}\right)$ and deuterium oxide $\left(\mathrm{D}_{2} \mathrm{O}\right)$ (Wako, degree of deuteration $>99.9 \%$ ). Before scattering and spectroscopic measurements, the solutions were kept at $5{ }^{\circ} \mathrm{C}$ for several days for equilibration.

Small- and wide-angle X-ray scattering (SWAXS) experiments in a scattering vector range of $0.07-28 \mathrm{~nm}^{-1}$ were carried out using a SAXSess camera (Anton Paar, Austria). Sample temperature was controlled with an accuracy of $\pm 0.1^{\circ} \mathrm{C}$. The obtained solution data were corrected for background scattering from the capillary cell and the solvent. To obtain scattering intensities equivalent to those measured by means of an ideal point collimation apparatus, a modelindependent collimation-correction procedure relying on the Lake algorithm [33] was performed. The measured scattering intensities were converted to absolute values by normalizing to the forward intensity determined for water [34]. As the pNIPAm in the $\mathrm{H}_{2} \mathrm{O}$ sample exhibited phase separation above $34{ }^{\circ} \mathrm{C}$, the rotor cell setup was used to prevent precipitation.
Spectra of the complex permittivity $\varepsilon^{*}(v)$ of aqueous pNIPAm solutions at identical $w$ were determined in the frequency range between 0.2 and $89 \mathrm{GHz}$ using a vector network analyzer (VNA) (Agilent, E8364B) with open-ended coaxial-line probes (Agilent, 85070E-020/-050) and $A$ - and $E$-band waveguide interferometers (Regensburg) [35]. The VNA instrument was calibrated with air, mercury, and water. Uncertainty of temperature control was $\pm 0.03{ }^{\circ} \mathrm{C}$.

\section{RESULTS AND DISCUSSION}

\section{A. Static structure}

Figures 1(a) and 1(b) show SWAXS intensities $I(q)$ of pNIPAm in $\mathrm{H}_{2} \mathrm{O}$ and $\mathrm{D}_{2} \mathrm{O}$ respectively as a function of temperature $T$. For the full description of $I(q)$, a sum of several scattering components is necessary [Figs. 1(c)-1(h)]. At low $T$, the lowest- $q$ portion of $I(q)$ can be entirely described by the Ornstein-Zernike (OZ) equation,

$$
I_{\mathrm{OZ}}(q)=\frac{I_{\mathrm{OZ}}(0)}{1+\xi^{2} q^{2}},
$$

where $\xi$ is the correlation length and $I_{\mathrm{OZ}}(0)$ is the forward intensity extrapolated to zero scattering vector. The OZ-type contribution reflects the fractal nature of the swollen coil polymer network [37]. With increasing $T$, the forward intensity increases, whereas simultaneously the shoulder exhibits a significant low- $q$ shift. Additionally, an excess contribution grows in at $q<0.2 \mathrm{~nm}^{-1}$ and becomes pronounced at $T>$ $32{ }^{\circ} \mathrm{C}$. This component can be formally described by the Guinier equation [38],

$$
I_{\mathrm{G}}(q)=I_{\mathrm{G}}(0) \exp \left[-\frac{\left(R_{g} q\right)^{2}}{3}\right]
$$


where $I_{\mathrm{G}}(0)$ and $R_{g}$ denote the asymptotic Guinier forward intensity and the radius of gyration, respectively. The emergence of this Guinier-type excess forward scattering even at $T<T_{\mathrm{S}}$ appears to indicate that already in the one phase region pNIPAm chains are in an aggregation regime [39].

At all temperatures, two broad interference peaks in the small, respectively wide, angle regime, hereafter called low- $q$ (L) and high- $q(\mathrm{H})$ peaks, can clearly be seen. These contributions, which were outside the reach of previous SANS studies $[17,18]$, are modeled by Lorentzian parts of the pseudo-Voigt equations [40],

$$
I_{\mathrm{V}_{x}}(q)=\frac{I_{\mathrm{V}_{x}}\left(q_{x}^{*}\right)}{1+\left(q-q_{x}^{*}\right)^{2} \xi_{x}^{*^{2}}}
$$

for $x=\mathrm{L}$ and $\mathrm{H}$, where $q_{x}^{*}$ denotes the peak position and $I_{\mathrm{V}_{x}}\left(q_{x}^{*}\right)$ is the maximum intensity at $q=q_{x}^{*}$; the sharpness parameter $\xi_{x}^{*}$ is related to the full width at half maximum (FWHM). The position of the high- $q$ peak, $q_{\mathrm{H}}^{*} \approx 15-16 \mathrm{~nm}^{-1}$, remains practically unchanged on variation of $T$. Judging from the corresponding length scale $(<0.5 \mathrm{~nm})$, this contribution originates from intrachain radial distributions. On the other hand, the emergence of the low- $q$ peak, centered at approximately 3-6 nm ${ }^{-1}$ depending on $T$, manifests the presence of an intermediate-range order, whose length scale is far larger than that of the radial distributions but somewhat smaller than or rather similar to that of the density fluctuations of the swollen polymer network. Note that conventional watersoluble random coil polymers, like dextran, often exhibit rod scattering in this $q$ range but does not show such an interference peak. Therefore, the temperature dependence of this peak deserves to be scrutinized as it should be the key to a better understanding of the phase behavior of pNIPAm.

Above $T_{\mathrm{S}}, I_{\mathrm{OZ}}(0)$ abruptly reduces whereas the forward intensity approaches the Porod scattering limit [38],

$$
I_{\mathrm{P}}(q)=K_{\mathrm{P}} q^{-4}
$$

where $K_{\mathrm{P}}$ is the Porod intensity. This indicates the collapse of the swollen polymer network and the formation of a sharp interface between polymer-rich and water-rich phases. We find a higher value for $K_{\mathrm{P}}$ in $\mathrm{D}_{2} \mathrm{O}$ than in $\mathrm{H}_{2} \mathrm{O}$, which is indicative of a greater surface area per unit volume in $\mathrm{D}_{2} \mathrm{O}$.

\section{B. Critical behavior}

Figure 2 shows $\xi$ and $I_{\mathrm{OZ}}(0)$ of the $\mathrm{OZ}$ contribution as a function of $T$. For semidilute solutions, $\xi$ should be interpreted as the mesh or blob size of the interpenetrating chains [37] whereas $I_{\mathrm{OZ}}(0)$ is related to the magnitude of the density fluctuation, being proportional to the osmotic compressibility [41]. We observe simultaneous divergence of $\xi$ and $I_{\mathrm{OZ}}(0)$ at the spinodal temperature $T_{\mathrm{S}}$. Below $T_{\mathrm{S}}$, these quantities are described by

$$
\xi(T)=\xi_{0}\left|\left(T-T_{\mathrm{S}}\right) / T_{\mathrm{S}}\right|^{-v}
$$

and

$$
I_{\mathrm{OZ}}(0, T)=I_{0}\left|\left(T-T_{\mathrm{S}}\right) / T_{\mathrm{S}}\right|^{-\gamma},
$$

where $\xi_{0}$ and $I_{0}$ are the bare correlation length and forward intensity, respectively, which are treated as $T$-independent constants. We obtain $T_{\mathrm{S}}=34.0^{\circ} \mathrm{C}$ in $\mathrm{H}_{2} \mathrm{O}$ while $T_{\mathrm{S}}=34.8^{\circ} \mathrm{C}$
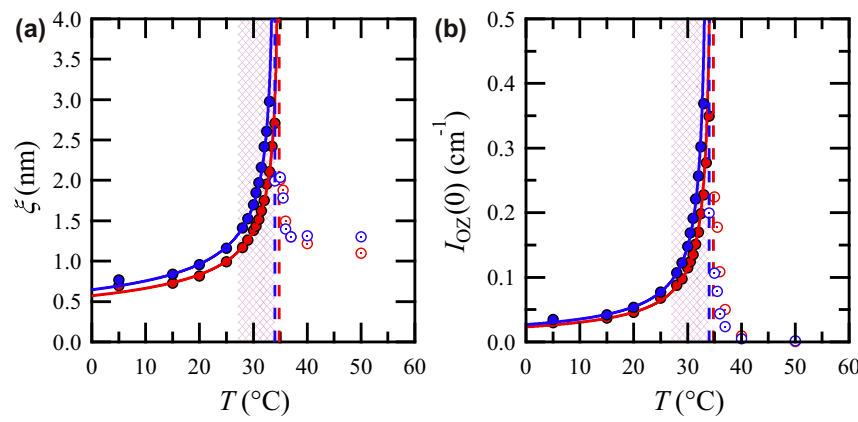

FIG. 2. Temperature dependence of (a) the correlation length $\xi$ and (b) the asymptotic $\mathrm{OZ}$ forward intensity $\mathrm{I}_{\mathrm{OZ}}(0)$ of semidilute pNIPAm solutions in $\mathrm{H}_{2} \mathrm{O}$ (blue) and $\mathrm{D}_{2} \mathrm{O}$ (red). Solid lines represent the power-law fits [Eqs. (5) and (6)] and vertical dashed lines highlight $T_{\mathrm{S}}$; open circles indicate the data for temperatures above $T_{\mathrm{S}}$.

in $\mathrm{D}_{2} \mathrm{O}$. This confirms that substitution of $\mathrm{D}_{2} \mathrm{O}$ for $\mathrm{H}_{2} \mathrm{O}$ as solvent leads to an increase of $T_{\mathrm{S}}$ by about $1 \mathrm{~K}$, consistent with the results of previous measurements of macroscopic properties [19-21]. The evaluated critical exponents are $v=$ 0.45 and $\gamma=0.80$ in $\mathrm{H}_{2} \mathrm{O}$ and $\nu=0.44$ and $\gamma=0.80$ in $\mathrm{D}_{2} \mathrm{O}$. Within experimental uncertainty, the values of $v$ and $\gamma$ are independent of the solvent conditions and systematically smaller than those predicted by mean-field theory $(v=0.5$ and $\gamma=1.0)$. The present critical exponents are in excellent agreement with those reported in the previous SANS study ( $v=0.44$ and $\gamma=0.81$ in $\mathrm{D}_{2} \mathrm{O}$ ) [18] despite the necessarily different choice of the fit model for the present $I(q)$ because of the extended $q$ range.

\section{Cooperative dynamics}

The hydration state of the pNIPAm chains in $\mathrm{H}_{2} \mathrm{O}$ is monitored by DRS. Figure 3 shows a temperature series of the complex permittivity spectra, $\varepsilon^{*}(v)=\varepsilon^{\prime}(v)-i \varepsilon^{\prime \prime}(v)$, of aqueous pNIPAm, where $\varepsilon^{\prime}(\nu)$ and $\varepsilon^{\prime \prime}(\nu)$ are the relative permittivity and dielectric loss, respectively. In the covered frequency range $(0.2-89 \mathrm{GHz})$, the dielectric spectrum of neat water is almost perfectly described by a single Debye-type relaxation function [42]. We find that well below $T_{\mathrm{S}}$, the addition of pNIPAm induces a marked low-frequency broadening of $\varepsilon^{\prime \prime}(v)$. With increasing $T$, the spectrum gets narrower. This effect becomes marked especially at $T>T_{\mathrm{S}}-6 \mathrm{~K}$.

We find that among the tested models, a superposition of two Debye relaxation functions,

$$
\varepsilon^{*}(\nu)=\frac{\Delta \varepsilon_{1}}{1+i 2 \pi \nu \tau_{1}}+\frac{\Delta \varepsilon_{2}}{1+i 2 \pi \nu \tau_{2}}+\varepsilon_{\infty},
$$

gives a consistent description of the experimental spectra at all investigated $T$. Here, the subscripts $j=1$ and 2 designate the low- and high-frequency relaxations respectively, which are characterized by their relaxation times $\tau_{j}\left(\tau_{1}>\tau_{2}\right)$ and amplitudes $\Delta \varepsilon_{j}$ (see Fig. 4). The loss peak frequency (and thus relaxation time $\tau_{2}$ ) of the high-frequency mode is found to be almost identical to that of pure water at all investigated $T$. Thus, this process is attributed to the cooperative rearrangement of the hydrogen-bond network of bulk water [42]. Its relaxation amplitude $\Delta \varepsilon_{2}$ reflects the concentration of water molecules behaving as bulklike water in solution. On the other hand, the 

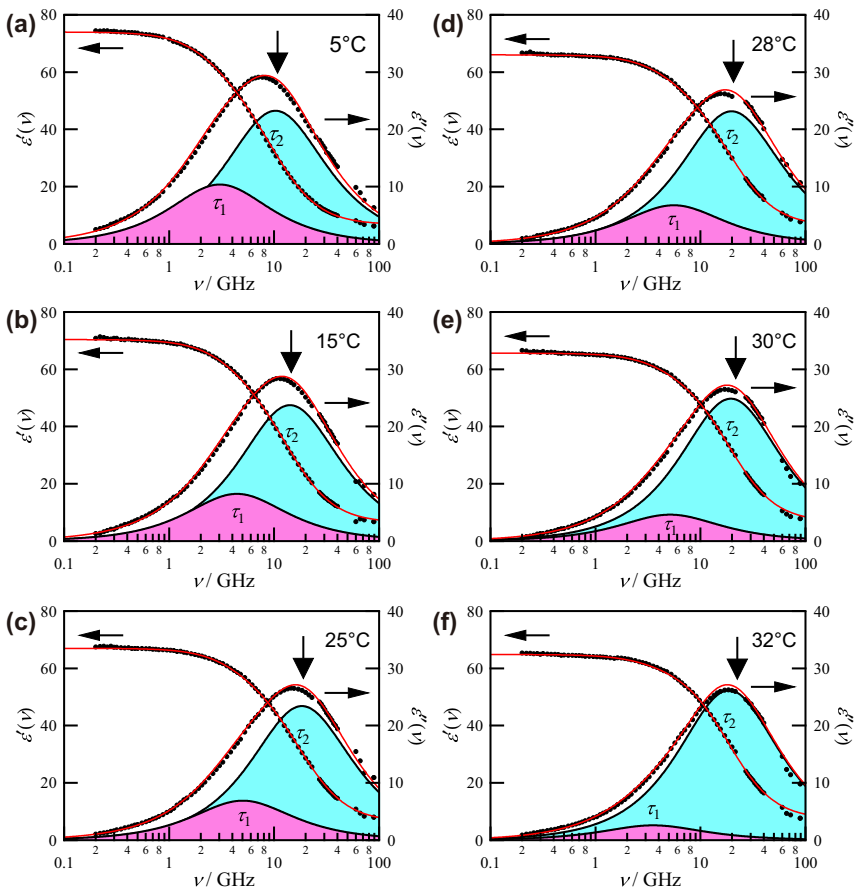

FIG. 3. Relative permittivity $\varepsilon^{\prime}(v)$ and dielectric loss $\varepsilon^{\prime \prime}(v)$ spectra of pNIPAm $(w=0.13)$ in $\mathrm{H}_{2} \mathrm{O}$ at (a) $5^{\circ} \mathrm{C}$, (b) $15^{\circ} \mathrm{C}$, (c) $25^{\circ} \mathrm{C}$, (d) $28^{\circ} \mathrm{C}$, (e) $30^{\circ} \mathrm{C}$, and (f) $32^{\circ} \mathrm{C}$. The red lines are the sum of two Debye relaxation functions [Eq. (7)]. The shaded areas represent the contributions of the resolved modes $(j=1$ and 2$)$. The vertical arrows indicate the loss peak frequency of neat water at identical $T$.

additional low-frequency mode $(j=1)$ reflects the retarded (slow) dynamics of $\mathrm{H}_{2} \mathrm{O}$ molecules in the hydration layer. Here, a contribution from dipolar pNIPAm side chains is possible but-as discussed below-apparently not significant. Segmental motions of the polymer and a possible MaxwellWagner-Sillars contribution of the collapsed polymer chain can also be neglected as these relaxations are in the megahertz region [29].

Below $28^{\circ} \mathrm{C}, \Delta \varepsilon_{1}$ shows a moderate decrease with $T$ while $\Delta \varepsilon_{2}$ remains nearly constant. However, increasing $T$ above $28^{\circ} \mathrm{C}$ leads to a pronounced decrease of $\Delta \varepsilon_{1}$ and a corresponding increase of $\Delta \varepsilon_{2}$. Such behavior implies that
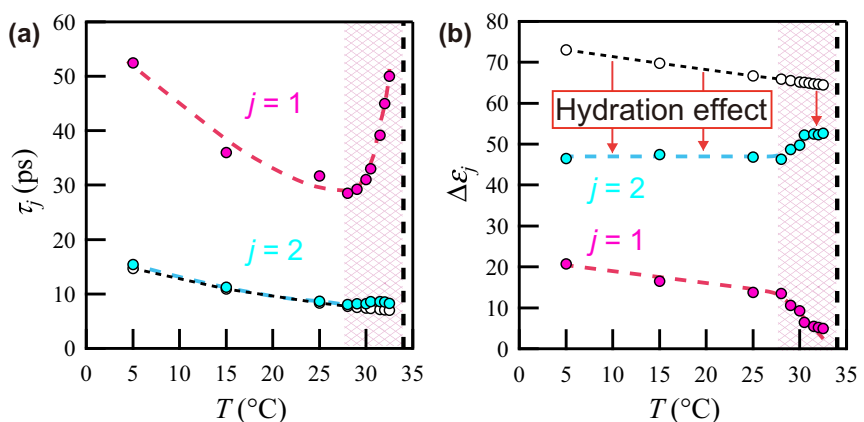

FIG. 4. (a) Relaxation times $\tau_{j}$ and (b) relaxation amplitudes $\Delta \varepsilon_{j}$ of the resolved modes (filled symbols). The empty symbols in (a) and (b) show the relaxation time and the concentration normalized amplitude of neat water, respectively. The vertical dotted lines highlight $T_{\mathrm{S}}$. on approaching $T_{\mathrm{S}}$, water molecules are released from the pNIPAm chains.

\section{Hydration/dehydration behavior}

To obtain deeper insights into the hydration state of the pNIPAm chains, we evaluate effective hydration numbers per monomer unit using the generalized Cavell equation [43-45],

$$
\Delta \varepsilon_{j}=\frac{\varepsilon}{\varepsilon+(1-\varepsilon) A_{j}} \frac{N_{\mathrm{A}}}{3 k_{\mathrm{B}} T \varepsilon_{0}} \frac{g_{j} \mu_{\mathrm{G}, j}^{2}}{\left(1-\alpha_{j} f_{j}\right)^{2}} c_{j},
$$

where $\Delta \varepsilon_{j}$ is the relaxation amplitude of a $j$ th process, $\varepsilon$ is the static permittivity, $A_{j}$ is the cavity-field factor, $N_{\mathrm{A}}$ is the Avogadro constant, $k_{\mathrm{B}}$ is the Boltzmann constant, $T$ is the temperature in kelvin, and $\varepsilon_{0}$ is the vacuum permittivity; $c_{j}$ is the molar concentration and $\mu_{\mathrm{G}, j}$ is the gas-phase dipole moment of the species causing process $j, \alpha_{j}$ is its polarizability, and $f_{j}$ is the corresponding reaction-field factor [46]. For water molecules $A_{j}=1 / 3$ (spherical shape) is assumed.

Normalization of Eq. (8) to the expression for neat water yields the apparent concentration $c_{j}(c)^{\text {app }}$ at solute concentration $c$ as

$$
c_{j}(c)^{\mathrm{app}}=\frac{\varepsilon(0)[2 \varepsilon(c)+1]}{\varepsilon(c)[2 \varepsilon(0)+1]} \frac{\left[1-\alpha_{\mathrm{w}} f_{\mathrm{w}}(c)\right]^{2}}{\left[1-\alpha_{\mathrm{w}} f_{\mathrm{w}}(0)\right]^{2}} \frac{\Delta \varepsilon_{j}(c)}{\Delta \varepsilon_{\mathrm{w}}(0)} c_{\mathrm{w}}(0) .
$$

Substitution of $\Delta \varepsilon_{2}$ for $\Delta \varepsilon_{j}$ in Eq. (9) yields the apparent bulk water concentration $c_{\mathrm{w}}(c)^{\text {app }}$. According to the definition, $c_{\mathrm{w}}(c)^{\text {app }}$ can be interpreted as the concentration of water molecules that retain dynamic properties virtually identical to those of pure water despite the presence of solute molecules (pNIPAm). The total hydration number $Z_{\text {total }}$ is thus defined as

$$
Z_{\mathrm{total}}=\frac{c_{\mathrm{w}}(c)^{\mathrm{ana}}-c_{\mathrm{w}}(c)^{\mathrm{app}}}{c}
$$

by converting the difference between $c_{\mathrm{w}}(c)^{\text {app }}$ and the analytical concentration of water $c_{\mathrm{w}}(c)^{\text {ana }}$ into the corresponding number of water molecules per pNIPAm repeat unit, where $c$ is the analytical concentration of the latter.

On the other hand, the apparent concentration of slow water $c_{\text {slow }}(c)^{\text {app }}$ can be calculated by substituting $\Delta \varepsilon_{1}$ for $\Delta \varepsilon_{j}$ into Eq. (9). The corresponding number of slow water molecules per NIPAm monomer unit is obtained as

$$
Z_{\text {slow }}=\frac{c_{\text {slow }}(c)^{\text {app }}}{c} .
$$

As mentioned before, side-chain dipoles of pNIPAm potentially contribute to $\Delta \varepsilon_{1}$. However, this should result in $Z_{\text {slow }}$ values that are significantly larger than the corresponding data for $Z_{\text {total }}$. According to Fig. 5 this is not the case, so that the difference between $Z_{\text {total }}$ and $Z_{\text {slow }}$ can be identified as the number of water molecules that are strongly (irrotationally) bound to the solute, $Z_{\mathrm{ib}}=Z_{\text {total }}-Z_{\text {slow }}$. The obtained hydration numbers $Z_{\text {total }}, Z_{\text {slow }}$, and $Z_{\text {ib }}$ are summarized in Fig. 5.

Up to $28^{\circ} \mathrm{C}, Z_{\text {total }}$ and $Z_{\text {slow }}$ show only a moderate decrease with $T$. However, above that threshold, both hydration numbers rapidly drop. In the identical $T$ range, $\xi$ and $I_{\mathrm{OZ}}(0)$ show a drastic increase (Fig. 2). This indicates that dehydration of the pNIPAm chains does not occur monotonously upon heating but proceeds abruptly above $T_{\mathrm{S}}-6 \mathrm{~K}$, implying a strong 


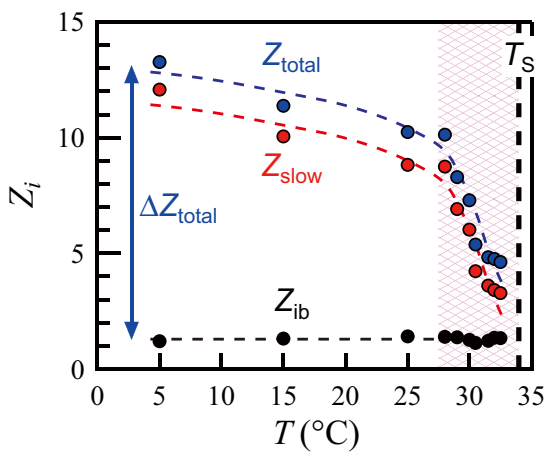

FIG. 5. Effective hydration numbers $Z_{i}$ per monomer unit of pNIPAm as a function of temperature. The total $Z_{\text {total }}$ and slow water $Z_{\text {slow }}$ hydration numbers are evaluated from $\Delta \varepsilon_{2}(T)$ and $\Delta \varepsilon_{1}(T)$, respectively. The difference, $Z_{\mathrm{ib}}=Z_{\text {total }}-Z_{\text {slow }}$, gives the corresponding number of apparently "frozen" (irrotationally bound) $\mathrm{H}_{2} \mathrm{O}$ molecules.

cooperative nature of the pNIPAm dehydration/hydration. The data also demonstrate that the dehydration is strongly coupled to the critical fluctuations of pNIPAm. We therefore infer that the exclusion of hydration water from the vicinity of the pNIPAm chains and the segregation of the system into nanometer-sized polymer-rich and water-rich domains occur in a concerted manner. This is also connected with a drastic slowdown of the hydration water dynamics, i.e., a strong rise of $\tau_{1}$ [Fig. 4(a)].

The practically constant value of $Z_{\mathrm{ib}} \approx 1.3$ probably means that these irrotationally bound water dipoles are not released from the pNIPAm chains even above $T_{\mathrm{S}}$. A similar number of strongly bound water molecules was also found for 1,3dimethylurea in a combined DRS and molecular dynamics study [47] and assigned to $\mathrm{H}_{2} \mathrm{O}$ dipoles strongly interacting with the carbonyl oxygen of the solute. This may also be the case for pNIPAm with its amide group in the side chains. Note that the change of the total hydration number upon phase transition, $\Delta Z_{\text {total }} \approx 12$, is in good agreement with the result obtained by thermodynamic measurements [48].

\section{E. Microscopic insights into the phase transition}

As shown in Figs. 1(g) and 1(h), well above $T_{\mathrm{S}}$, the measured intensities at $q<8 \mathrm{~nm}^{-1}$ can be approximated as $I_{\mathrm{P}}(q)+I_{\mathrm{V}_{\mathrm{L}}}(q)$. This means that the polymer-rich phase is virtually homogeneous on a length scale exceeding that of an intermediate-range order $\left(\sim 1 / q_{\mathrm{L}}^{*}\right)$, while on a shorter length scale, the system exhibits marked density fluctuations. This unambiguously demonstrates that the low- $q$ peak originates from intrinsic density fluctuations in the globule state of pNIPAm. From the emergence of a considerably broader but otherwise similar interference peak at temperatures far below $T_{\mathrm{S}}$, we argue that already in the one-phase region, an equivalent exists to the positional correlations between high-density scattering objects that characterize the globule state.

A comparable low- $q$ peak was observed for pNIPAm-based gels [49-51]. Our data firmly confirm that the emergence of this low- $q$ peak is not a gel specific phenomenon but an intrinsic property of the pNIPAm chain. Chalal et al. claimed that the peak observed for pNIPAm-based cryogels is due
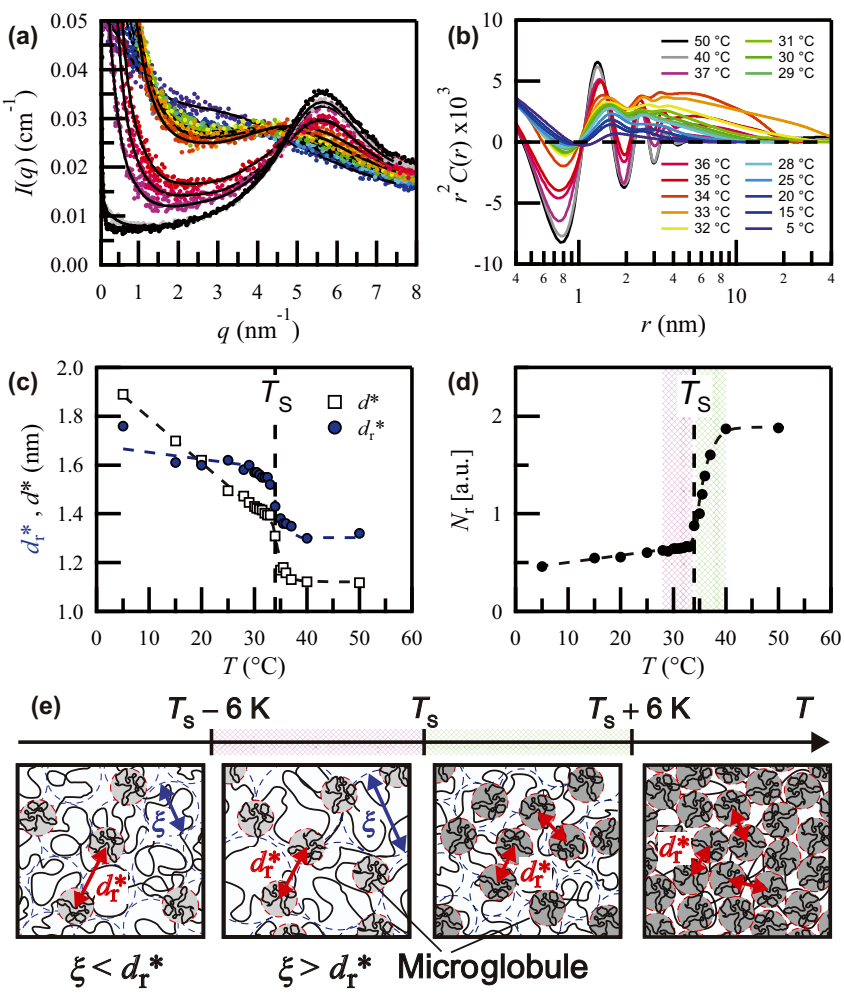

FIG. 6. Microglobule formation as revealed by SWAXS. (a) Absolute scattering intensity $I(q)$ for scattering vectors $q \leqslant 8 \mathrm{~nm}^{-1}$ and temperatures $5 \leqslant T /{ }^{\circ} \mathrm{C} \leqslant 50$ (symbols) and fits to $I(q)$ with an indirect Fourier transformation technique (lines). (b) Corresponding density autocorrelation functions $r^{2} C(r)$ of pNIPAm in $\mathrm{H}_{2} \mathrm{O}$ calculated from the $I(q)$ curves shown in (a). Temperature dependence of (c) the characteristic distance obtained by applying Bragg's law $\left(d^{*}=2 \pi / q_{\mathrm{L}}^{*}\right.$, empty square) and directly read out from the first (lowest- $r$ ) peak position in $r^{2} C(r)\left(d_{\mathrm{r}}^{*}\right.$, blue filled circle) and (d) the relative local number densities of the microglobules, $N_{\mathrm{r}} \propto \xi_{\mathrm{L}}^{*} / d_{\mathrm{r}}^{*}$. Those in $\mathrm{D}_{2} \mathrm{O}$ solvent are provided in the Supplemental Material [36]. (e) Schematic representation of the spatial density fluctuations of pNIPAm at different $T$ values. Far below $T_{\mathrm{S}}$, where $d_{\mathrm{r}}^{*}>\xi$, longer-range positional correlations between microglobules yield a broad interference peak. In the range of $T_{\mathrm{S}}-6 \mathrm{~K} \leqslant T \leqslant T_{\mathrm{S}}$, $\xi$ rapidly increases, leading to $\xi>d_{\mathrm{r}}^{*}$. For $T>T_{\mathrm{S}}$, the density fluctuation arising from the random coil chains rapidly disappears and the local number density of the microglobules shows a steplike increase. The condensation of the microglobules is completed at $T_{\mathrm{S}}+6 \mathrm{~K}$ and the polymer-rich phase can be understood as a high-density assembly of the microglobules.

to local packing arising from the hydrophobic association of isopropyl groups of two neighbor chains or from hydrogen bonds between amide groups [49]. However, this explanation cannot apply to pNIPAm solutions because for $T<28^{\circ} \mathrm{C}$, the values of the characteristic distance $d^{*}$, approximated as $2 \pi / q_{\mathrm{L}}^{*}\left(1.9 \mathrm{~nm}\right.$ at $5^{\circ} \mathrm{C}$ and $1.5 \mathrm{~nm}$ at $\left.25^{\circ} \mathrm{C}\right)$, certainly exceed the corresponding correlation lengths $\xi(0.77$ and $1.2 \mathrm{~nm})$ [Figs. 2(a) and 6(c)].

We are aware that the calculation of the characteristic distance from Bragg's law may be problematic for broad peaks. To overcome these issues, we calculate density autocorrelation functions (ACFs), $C(r)$, using an indirect Fourier 
transformation (IFT) technique [38] [Fig. 6(a)], where $C(r)$ is given by the inverse Fourier transformation of $I(q)$ as

$$
I(q)=4 \pi \int r^{2} C(r) \frac{\sin (q r)}{q r} d r .
$$

The resulting ACFs in the expression of $r^{2} C(r)$ are shown in Fig. 6(b). The low- $q$ peak is mapped into oscillations in $r^{2} C(r)$ and the characteristic distance in real space $d_{\mathrm{r}}^{*}$ can be directly taken from the first peak position [Fig. 6(c)]. For $T<T_{\mathrm{S}}, d^{*}$ monotonously decreases with $T$ from $1.9 \mathrm{~nm}$ at $5{ }^{\circ} \mathrm{C}$ to 1.4 $\mathrm{nm}$ at $33^{\circ} \mathrm{C}$ (just below $T_{\mathrm{S}}$ ) while $d_{\mathrm{r}}^{*} \approx 1.6 \mathrm{~nm}$ remains nearly constant, showing a gentler slope than $d^{*}$. Above $T_{\mathrm{S}}$, both $d^{*}$ and $d_{\mathrm{r}}^{*}$ drop significantly and converge to 1.1 and $1.3 \mathrm{~nm}$, respectively. Despite the somewhat different $T$ dependence of $d^{*}$ and $d_{\mathrm{r}}^{*}$, the major point that $\xi$ exceeds $d_{\mathrm{r}}^{*}$ only above approximately $28^{\circ} \mathrm{C}$ is unchanged. These observations lead us to conclude that above $T_{\mathrm{S}}$, the low- $q$ peak arises from positional correlations between the microglobules and that similar microglobules exist already in the one-phase region $\left(T<T_{\mathrm{S}}\right)$, as predicted in the cooperative hydration picture $[14,15]$.

Generally, the sharpness of an interference peak is related to the size of organized domains. Using FWHM, $\Delta \beta=2 / \xi_{\mathrm{L}}^{*}$, the domain size $D$ is given by $D=2 \pi / \Delta \beta$. By extending a crystallographic method, the (relative) local number density of the microglobules may be deduced as $N_{\mathrm{r}} \propto D / d_{\mathrm{r}}^{*}$ [Fig. 6(d)]. The values of $N_{\mathrm{r}}$ exhibit only a moderate increase for $T<T_{\mathrm{S}}$, but show a steplike jump just above $T_{\mathrm{S}}$, reaching a plateau at $T>40^{\circ} \mathrm{C}$. In Figs. 6(d) and 6(e), the characteristic $T$ ranges of the divergence of $\xi$ attaining $\xi>d_{\mathrm{r}}^{*}$ and the rapid condensation of the microglobules are highlighted in red and green colors, respectively. We stress the fact that the condensation of the microglobules does not occur in parallel with the divergence of $\xi$. In $T_{\mathrm{S}}<T<T_{\mathrm{S}}+6 \mathrm{~K}$, where the swollen polymer networks are mostly collapsed, the microglobules rapidly condense into a high-density state as $N_{\mathrm{r}}$ exhibits a steplike increase. These findings demonstrate that the phase transition of pNIPAm in aqueous media can be viewed as a transition between a state of low density of the microglobules to a state with high microglobule density [Fig. 6(d)]. Accordingly, we can now identify the local number density of the microglobules as an additional microscopic order parameter monitoring the phase transition [Fig. 6(e)].

\section{CONCLUSIONS}

To summarize, we have presented experimental evidence that critical fluctuations and dehydration of the pNIPAm chains are closely coupled, due to the strong cooperative nature of the dehydration/hydration of the polymer chains. We have also shown similarity and substantial difference of the critical fluctuation of pNIPAm in $\mathrm{H}_{2} \mathrm{O}$ and $\mathrm{D}_{2} \mathrm{O}$, confirming about $1 \mathrm{~K}$ higher $T_{\mathrm{S}}$ in $\mathrm{D}_{2} \mathrm{O}$ and almost identical critical exponents independent of the solvent conditions. The data demonstrate the onset of microglobule formation far below $T_{\mathrm{S}}$, where the long-range positional correlations between these microglobules $\left(d_{\mathrm{r}}^{*}>\xi\right)$ give a broad interference peak.

Above $T_{\mathrm{S}}$ the polymer-rich phase is characterized by a high number density state of these microglobules. For $T>$ $T_{\mathrm{S}}-6 \mathrm{~K}, \xi$ rapidly increases, resulting in $\xi>d_{\mathrm{r}}^{*}$. For $T>T_{\mathrm{S}}$, the density fluctuation arising from the random coil chains rapidly disappears, whereas the local number density of the microglobules shows a steplike increase.

Importantly, we find that the condensation of the microglobules does not occur simultaneously with the divergence of $\xi$. Instead, the condensation sets in just above $T_{\mathrm{S}}$ and is completed at temperatures about $6 \mathrm{~K}$ above $T_{\mathrm{S}}$ whereas $\xi$ already diverges when approaching $T_{\mathrm{S}}$. Therefore, the phase transition of aqueous pNIPAm solution can be understood as a transition from a low-density to a high-density state of the microglobules. Besides the ordinary density order parameter, whose spatial fluctuations are manifested by $\xi$, the local number density of the microglobules can be identified as a microscopic order parameter governing the phase transition of aqueous pNIPAm solution. The change in solvent water to a weaker hydrogen bond structure above the cloud temperature, claimed by a recent Raman-MCR study [32], may be associated with the completion of the high-density microglobule assemblies.

We note that structural changes related to the volume-phase transition (VPT) of pNIPAm-based gels are often regarded as gel-specific phenomena, but this is probably not correct. As we have shown, many of these changes arise essentially from an intrinsic property of the pNIPAm chain, namely the temperature-induced change of the interchain interactions and the mixing scheme of pNIPAm in aqueous environment. We anticipate that this work has a potential impact on subsequent investigation into effects of coexisting alcohols $[15,16,31]$ and ions on the phase transition of pNIPAm and all kinds of other thermoresponsive polymers in aqueous media.

\section{ACKNOWLEDGMENTS}

The authors wish to thank T. Miyashita, A. Nazet, and Dr. T. Sonnleitner for their help with SWAXS and DRS measurements. We are also grateful to Prof. Y. Katsumoto (Fukuoka University) and Dr. A. Chiba (Keio University) for their stimulating discussion. K.Y. acknowledges a research fellowship grant-in-aid of the Japan Society for the Promotion of Science (Grant No. 17J06213).
[1] D. Chandler, Nature (London) 437, 640 (2005).

[2] T. Tanaka, Phys. Rev. Lett. 40, 820 (1978).

[3] Y. Hirokawa and T. Tanaka, J. Chem. Phys. 81, 6379 (1984).

[4] K. A. Dill, Biochemistry 29, 7133 (1990).

[5] M. Heskins and J. E. Guillet, J. Macromol. Sci., Pure Appl. Chem. 2, 1441 (1968).
[6] V. Aseyev, H. Tenhu, and F. M. Winnik, in Self Organized Nanostructures of Amphiphilic Block Copolymers II (Springer, Berlin, Heidelberg, 2011), pp. 29-89.

[7] A. Halperin, M. Kröger, and F. M. Winnik, Angew. Chem., Int. Ed. 54, 15342 (2015).

[8] K. Kubota, S. Fujishige, and I. Ando, J. Phys. Chem. 94, 5154 (1990). 
[9] C. Wu and S. Zhou, Macromolecules 28, 8381 (1995).

[10] C. Wu and S. Zhou, Macromolecules 28, 5388 (1995).

[11] C. Wu and X. Wang, Phys. Rev. Lett. 80, 4092 (1998).

[12] X. Wang, X. Qiu, and C. Wu, Macromolecules 31, 2972 (1998).

[13] P. R. ten Wolde and D. Chandler, Proc. Natl. Acad. Sci. USA 99, 6539 (2002).

[14] Y. Okada and F. Tanaka, Macromolecules 38, 4465 (2005).

[15] F. Tanaka, T. Koga, and F. M. Winnik, Phys. Rev. Lett. 101, 028302 (2008).

[16] I. Bischofberger, D. Calzolari, P. De Los Rios, I. Jelezarov, and V. Trappe, Sci. Rep. 4, 4377 (2014).

[17] M. Shibayama, T. Tanaka, and C. C. Han, J. Chem. Phys. 97, 6829 (1992).

[18] A. Meier-Koll, V. Pipich, P. Busch, C. M. Papadakis, and P. Müller-Buschbaum, Langmuir 28, 8791 (2012).

[19] X. Wang and C. Wu, Macromolecules 32, 4299 (1999).

[20] H. Shirota, N. Kuwabara, K. Ohkawa, and K. Horie, J. Phys. Chem. B 103, 10400 (1999).

[21] P. Kujawa and F. M. Winnik, Macromolecules 34, 4130 (2001).

[22] T. Tokuhiro, T. Amiya, A. Mamada, and T. Tanaka, Macromolecules 24, 2936 (1991).

[23] Y. Maeda, T. Higuchi, and I. Ikeda, Langmuir 16, 7503 (2000).

[24] Y. Ono and T. Shikata, J. Am. Chem. Soc. 128, 10030 (2006).

[25] M. J. N. Junk, U. Jonas, and D. Hinderberger, Small 4, 1485 (2008).

[26] Z. Ahmed, E. A. Gooding, K. V. Pimenov, L. Wang, and S. A. Asher, J. Phys. Chem. B 113, 4248 (2009).

[27] Y. Satokawa, T. Shikata, F. Tanaka, X.-p. Qiu, and F. M. Winnik, Macromolecules 42, 1400 (2009).

[28] M. Füllbrandt, E. Ermilova, A. Asadujjaman, R. Hölzel, F. F. Bier, R. von Klitzing, and A. Schönhals, J. Phys. Chem. B 118, 3750 (2014).

[29] M. Yang, C. Liu, and K. Zhao, Phys. Chem. Chem. Phys. 19, 15433 (2017).

[30] M. Yang, W. Su, and K. Zhao, J. Polym. Sci., Part B: Polym. Phys. 55, 1859 (2017).

[31] M. Yang and K. Zhao, J. Polym. Sci., Part B: Polym. Phys. 55, 1227 (2017).

[32] K. Mochizuki and D. Ben-Amotz, J. Phys. Chem. Lett. 8, 1360 (2017).

[33] J. A. Lake, Acta Crystallogr. 23, 191 (1967).

[34] D. Orthaber, A. Bergmann, and O. Glatter, J. Appl. Crystallogr. 33, 218 (2000).
[35] T. Sonnleitner, D. A. Turton, S. Waselikowski, J. Hunger, A. Stoppa, M. Walther, K. Wynne, and R. Buchner, J. Mol. Liq. 192, 19 (2014).

[36] See Supplemental Material at http://link.aps.org/supplemental/ 10.1103/PhysRevMaterials.2.085601 for additional information on the temperature-dependent scattering intensities of pNIPAm in $\mathrm{D}_{2} \mathrm{O}$ solvent and micro-globule formation of pNIPAm in $\mathrm{D}_{2} \mathrm{O}$ solvent.

[37] P.-G. de Gennes, Scaling Concepts in Polymer Physics (Cornell University Press, Ithaca, 1979).

[38] O. Glatter and O. Kratky, Small Angle X-Ray Scattering (Academic, London, 1982).

[39] K. Nishi, T. Hiroi, K. Hashimoto, K. Fujii, Y.-S. Han, T.-H. Kim, Y. Katsumoto, and M. Shibayama, Macromolecules 46, 6225 (2013).

[40] G. K. Wertheim, M. A. Butler, K. W. West, and D. N. E. Buchanan, Rev. Sci. Instrum. 45, 1369 (1974).

[41] G. R. Strobl, The Physics of Polymers: Concepts for Understanding Their Structures and Behavior (Springer, Berlin, Heidelberg, 2007).

[42] T. Fukasawa, T. Sato, J. Watanabe, Y. Hama, W. Kunz, and R. Buchner, Phys. Rev. Lett. 95, 197802 (2005).

[43] E. A. S. Cavell, P. C. Knight, and M. A. Sheikh, Trans. Faraday Soc. 67, 2225 (1971).

[44] R. Buchner, C. Hölzl, J. Stauber, and J. Barthel, Phys. Chem. Chem. Phys. 4, 2169 (2002).

[45] A. Eiberweiser, A. Nazet, G. Hefter, and R. Buchner, J. Phys. Chem. B 119, 5270 (2015).

[46] R. Buchner, S. G. Capewell, G. Hefter, and P. M. May, J. Phys. Chem. B 103, 1185 (1999).

[47] V. Agieienko, D. Horinek, and R. Buchner, Phys. Chem. Chem. Phys. 19, 219 (2017).

[48] M. Shibayama, M. Morimoto, and S. Nomura, Macromolecules 27, 5060 (1994).

[49] M. Chalal, F. Ehrburger-Dolle, I. Morfin, F. Bley, M.-R. Aguilar de Armas, M.-L. López Donaire, J. San Roman, N. Bölgen, E. Pişkin, O. Ziane, and R. Casalegno, Macromolecules 43, 2009 (2010).

[50] P. Perez, F. Plieva, A. Gallardo, J. San Roman, M. R. Aguilar, I. Morfin, F. Ehrburger-Dolle, F. Bley, S. Mikhalovsky, I. Y. Galaev, and B. Mattiasson, Biomacromolecules 9, 66 (2008).

[51] T. Kureha, T. Sato, and D. Suzuki, Langmuir 30, 8717 (2014). 